\title{
Health Related Quality of Life (HRQOL) Measures in Healthcare Delivery System: Indian Perspective
}

\author{
Manthan Dilipkumar Janodia \\ Department of Pharmacy Management, Manipal College of Pharmaceutical Sciences, Manipal University, Manipal 576104, Karnataka, INDIA.
}

\begin{abstract}
Health Related Quality of Life (HROOL) studies are increasingly becoming common in developed countries to support product label claims. A large body of research is devoted to developing and validating $\mathrm{HRQOL}$ measures. Generic as well as disease specific instruments to measure HROOL are developed. Development and utilization of HROOL instruments increased during last decade with efforts to improve patient health and value of health care services. Physical, social and emotional impact of diseases and their impact on patients' lives are focus of the HROOL studies. HROOL, as a concept, differs from physiologic aspects of disease and has been recognized as important clinical outcome in studies that evaluates pharmaceutical agents, medical technology and healthcare. Regulatory agencies life Food and Drug Administration (FDA) and European Medicines Agency (EMEA) have accepted importance of $\mathrm{HRQOL}$ as valid measure in clinical trials to support product label claim. The use of HRQOL instruments have been predominantly confined to North America and Europe. Significant progress is not made regarding use of $\mathrm{HROOL}$ instruments in designing health policy in developing countries, including India. It is important to know how patients view their health and what their expectations from the
\end{abstract}

healthcare system are for designing appropriate health policy and its implementation, budget allocation and deployment of healthcare resources. India, with its vast population and increasing healthcare delivery challenges, should aim at creating infrastructure for carrying out $\mathrm{HROOL}$ studies and use of Patient Reported Outcome (PRO) instruments in making and implementing health policy decisions.

Key words: $\mathrm{HROOL}, \mathrm{QOL}$, Healthcare Delivery, PRO, India.

Correspondence :

Manthan D Janodia,

Associate Professor, Department of Pharmacy Management,

Manipal College of Pharmaceutical Sciences, Manipal University,

Manipal 576104, Karnataka, INDIA.

Phone: 9880130878

E-mail: manthan.janodia@gmail.com

DOI: 10.5530/jyp.2016.3.1

\section{INTRODUCTION}

Health is fundamental to human life. Within healthcare systems, health related quality of life (HRQOL) studies are becoming increasingly common in recent times. ${ }^{1}$ The World Health Organization (WHO), in its constitution, has defined health as "A state of complete physical, mental, and social well-being not merely the absence of disease or infirmity". ${ }^{2}$ This definition of health by WHO recognizes the importance of health measures beyond traditional clinical outcomes of morbidity and mortality., ${ }^{2,3}$ According to Centers for Disease Control and Prevention (CDC), measures of HRQOL has evolved since 1980 and include those aspects of Quality of Life (QOL) that can be clearly shown to affect health-either physical or mental. ${ }^{4}$ Leading health organizations has also identified HRQOL as a goal for people across all life stages and is a matter of concern among policymakers, researchers and healthcare practitioners. ${ }^{5}$ A large body of research has been devoted to development of HRQOL measures. ${ }^{6}$ Use of HRQOL instruments have been incorporated in health surveillance and are considered valid indicators for health needs, policy documents, service requirements and intervention outcomes. ${ }^{4}$ Development and utilization of HRQOL instruments increased in last decade in efforts to improve patient health and value of health care services. ${ }^{7}$ For many medical conditions and problems, it is possible to use a single measure to assess effectiveness, eg. blood pressure or a diagnostic test, but these approaches may not be suitable for comparing relative output of different interventions for different disease groups in various populations. ${ }^{8}$ The use of HRQOL studies is suitable in understanding effect of interventions on different populations. Physical, social and emotional impact of diseases and their impact on patients' lives are focus of HRQOL studies. HRQOL, as a concept, differs from physiologic aspects of disease and has been recognized as important clinical outcome in studies evaluating pharmaceutical agents, medical technology and healthcare. ${ }^{9}$
Health is interchangeably referred through three different concepts viz. Health Status, functional status and quality of life (QOL). The domain of health encompasses negative aspects of life such as death or disability as well as positive aspects such as happiness or role function. HRQOL studies are particularly useful in assessing chronic diseases. ${ }^{10}$ It is argued that valid and reliable measures assessing functional status and well-being of an individual may provide more detailed information about the health of individuals in population than through gross measures such as life expectancy and infant mortality. ${ }^{11}$ The measures and instruments used to evaluate HRQOL include generic instruments, with a fixed set of items regardless of disease state, and disease specific tools to address impact of certain disease. ${ }^{12}$ These instruments, both generic and disease specific, are developed in past 50 years. These instruments were single item global ratings initially, changed to more lengthy and extensive surveys of well-being and transformed into brief evaluations carried out through psychometric reduction methodologies. ${ }^{12}$

\section{QOL and HRQOL}

According to Cummins ${ }^{13}$ three major branches of science-Economics, Medicine and Social Sciences-currently uses concept of Quality of Life (QOL), leading to differing views on conceptualization and measurement of QOL. QOL has four conceptualization principles, which include:

a. QOL is multidimensional and is influenced by personal and environmental factors and their interaction

b. has the same component for all people

c. has both subjective and objective component

d. is enhanced by self determination, resources, purpose in life and a sense of belongingness ${ }^{13}$ 
QOL is a broad ranging concept and there is no universally accepted definition of QOL $^{14}$ QOL is perceived as a state of well-being. World Health Organization (WHO) defines QOL as "individuals perception of their position in life in the context of the culture and value systems in which they live in relation to their goals, expectations, standards and concerns". ${ }^{15}$ QOL gained prominence in social research studies since 1970s. Though frequently used, QOL is used interchangeably with other terms and phrases such as "life satisfaction", "functional statuses" and "well-being". 16

Since QOL has multidimensional aspects, it is neither feasible and practical nor, perhaps, possible to measure all aspects that are a part of broad concept of QOL. ${ }^{14}$ The inherent weakness of the concept of quality of life is that it is applied beyond the realms of health. ${ }^{8}$ The widely valued aspects of life, not generally considered "health" exist and include income, freedom, quality of the environment, culture, religion etc. ${ }^{17}$ This broad definition of QOL prompted researchers to develop and validate instruments that specifically measure health as an indicator of well-being and not merely Quality Of Life. The primary application of HRQOL measures is clinical evaluation but it has also been applied to health planning, population monitoring, health service research and policy evaluation. ${ }^{12}$ One potential limitation of biomedical measures is that it may not, sometimes, indicate improvement in health function and status. Incorporation of HRQOL measure to assess physical, psychological and social functioning complements the outcome and assessment derived from biomedical measures. ${ }^{18}$ Vitality, pain and cognitive function are also important domains of HRQOL. ${ }^{16}$ Generic instruments are applied to measure HRQOL across various health conditions. Disease specific instruments are used to measure a specific disease or group of diseases. ${ }^{19}$ Growing body of literature suggest increasing importance of HRQOL as an outcome measure ofclinical trials. ${ }^{20}$ Many randomized clinical trials often evaluate HRQOL as valid and useful endpoints in assessing morbidity and mortality in addition to traditional clinical outcomes. ${ }^{21}$

HRQOL is usually measured with "instruments", which refers to patient questionnaires. HRQOL instruments are classified as either generic or disease specific. The choice of HRQOL instrument depends on the research question being investigated and hypothesis that is tested in clinical trials. The purpose of using an instrument should be clearly stated as to assure that the questionnaire used is in accordance as desired and intended by developers of the instruments. ${ }^{22}$ A large body of HRQOL instruments, generic as well as disease specific, are developed over the years that measure aspects of well-being, pain, emotional distress, impact on daily activity, to name a few. PROs have become crucial endpoint in clinical studies over the years. Regulatory agencies in the US and Europe released guidelines for development and use of PROs. The guidelines aim to standardize quality standards and minimum requirements for PRO instruments. ${ }^{23}$

The issue of HRQOL, however, has limitations. As Gurkova ${ }^{24}$ has mentioned, debate around definition of HRQOL centers upon following issues:

a. The relationship and distinction between "health related quality of life" and "quality of life"

b. The relationship and distinction between "health" and "quality of life"

c. Distinction between indicators and HRQOL predictor, or between indicator and causal variables of QOL. ${ }^{24}$

Despite limitations, general consensus regarding HRQOL instruments include following attributes: ${ }^{24}$

a. HRQOL is multidimensional

b. HRQOL is subjective and value based

c. HRQOL is dynamic

d. HRQOL is defined in terms of perceived status e. HRQOL involves the individuals' perception of both positive and negative dimensions.

\section{HRQOL AND PRO}

As HRQOL measures patients' self assessment or important aspects of their well-being that are currently affected by current disease or treatment, use of Patient Reported Outcomes (PROs) have increased significantly. ${ }^{25}$ European Medicines Agency's (EMEA) Committee for Medicinal Products for Human Use (CHMP) published a reflection paper in July 2005 on the regulatory guidance for the use of Health Related Quality of Life (HRQOL) measures in the evaluation of medicinal products. ${ }^{25}$ The reflection paper discussed how HRQOL may have a place in drug evaluation process with the existing guidance documents and stated that approval of a new medicinal product is based on its efficacy and safety in given condition, through established endpoints concerning core symptoms and condition. The paper also stated that HRQL assessment is optional and needs to be supported by data collected with the help of validated instruments. ${ }^{26}$

In February 2006, US Food and Drug Administration (FDA) published its draft guidance on "Patient Reported Outcome Measures: Use in medical product development to support labeling claim". ${ }^{27}$ Since FDA published only a guidance document, reasonable deviations to approaches outlined in the guidance may be acceptable with documented justification..$^{28}$ However, draft guidance by FDA is considered milestone for acknowledging the importance of PRO measures in clinical trials. ${ }^{29}$ The approach adopted by EMEA is more flexible and general whereas FDA provides more direct recommendations. The draft guidance by FDA had several limitations in the use of PRO measures during clinical development and trials of medicinal products. ${ }^{29,30}$ These limitations and suggested measures to overcome it are discussed by Revicki ${ }^{29}$ and, Arpinelli and Bamfi. ${ }^{30}$ In the revised FDA guidance released in 2009, the agency has clarified that PRO data can be used to measure the risk of treatment as well as benefit, and the development of PRO measures is an iterative process with no single correct approach. In the final guidance document, emphasis placed by FDA is on defining the role of a PRO endpoint in clinical trials and documenting development history of a PRO. ${ }^{31}$

\section{Use of HRQOL and PRO in India}

The extensive research carried out using HRQOL measures in North America and Europe is not evident in developing countries, including India a computerized search was carried out for published literature regarding, primarily, Health Related Quality of Life (HRQOL) and to a lesser extent to "Quality of Life (QOL) studies in India. Pub Med data was used to extract studies related to HRQOL and QOL in India. The search terms used were "HRQOL" and "India". The search was restricted for a time period from January 01, 2001 to August 31, 2013. Another search term "SF-36", in addition to above search terms "HRQOL" and "India" was added to search and was restricted for time period mentioned above. The search term "SF-36" was added as "SF-36" is 36 item Short Form Health Survey. SF - 36 is a generic and coherent measure, administered to adult patients to measure their Quality of Life (QOL) through self reporting.

A total of 61 studies were found for search terms "HRQOL" and "India". Out of these 61 studies found using the search terms, 11 studies were carried out in settings/population outside India, thus the number of HRQOL studies pertaining to India trickled down to 50, which is significantly less than the studies carried out in North America and Europe. The search strategy neither focused on the quality of studies nor the type of studies and disease conditions for which HRQOL measures are used. It was observed from the search data that published HRQOL and QOL studies within India and/or by Indian researchers were primarily 
after 2005. A large number of studies are published after 2010; during 2005-2009 the number of studies related to HRQOL was handful, totaling 13 whereas after 2010 the number of studies published on HRQOL within Indian setting rose substantially. Studies related to HRQOL, published in Indian setting and/or by Indian researchers, during the last four years from 2010-2013, rose to 37. This clearly shows interest among Indian researchers to conduct more research in this field. The use of search terms "HRQOL", "India" and "SF-36" yielded only 14 studies out of which 7 studies were carried out in settings outside India.

India has unique challenges in delivering healthcare due to its huge population size and growing number of elderly population. ${ }^{32}$ The results obtained through combining different search terms, show that effective use of HRQOL and PRO measures is in nascent stage in India. The use of Patient Reported Outcome (PRO) measures may help in assessing impact of healthcare interventions on patients, assist in resource allocation, evaluate effects of change to services and assist clinical governance.

In order to bring new technology as well as approaches, and innovations into public health system, ${ }^{32}$ the research regarding use of HRQOL and/or PRO measures, to identify gaps in healthcare resource allocation is essential. The use of HRQOL and PRO measures may help plug gaps in healthcare delivery and resource allocation effectively and efficiently in India.

\section{SUMMARY}

HRQOL and PRO measures are increasingly becoming common in healthcare decision making. Over the years, the tools of HRQOL and PRO are developed and its validity and reliability is well established. Regulatory agencies are increasingly favoring use of HRQOL measures during clinical trials as secondary endpoints during drug approval process. It is essential to understand viewpoint of recipients of healthcare system and equally important to integrate it into decision making that would lead to rational budget allocation, deployment of healthcare resources and access to these services. India with its vast population and increasing healthcare challenges in its delivery, should aim at creating infrastructure for carrying out HRQOL studies and use of PRO instruments in making health policy decisions. The objectives of providing Universal Health Coverage seem unlikely, if HRQOL and PRO measures are not integrated with clinical measures of health outcomes.

\section{ACKNOWLEDGMENT}

University Grants Commission, New Delhi, India for Raman Post Doctoral Fellowship at Department of Pharmacy Administration, School of Pharmacy, University of Mississippi, Mississippi, USA.

\section{CONFLICT OF INTEREST}

The author declares no conflict of interest.

\section{AUTHORS' CONTRIBUTION}

MJ conceptualized the idea and conceived the study, collected literature and data, and drafted the manuscript.

\section{ABOUT AUTHOR}

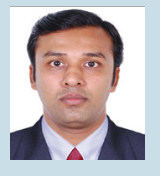

Manthan D Janodia: Is Associate Professor in Department of Pharmacy Management, Manipal College of Pharmaceutical Sciences, Manipal University. He teaches pharmaceutical management to undergraduate students and intellectual property rights to post graduate students of Pharmacy. He completed his PhD in the field of Intellectual Property Rights from Manipal University in 2009. He received Raman Post Doctoral Fellowship to carry out PDF at Department of Pharmacy Administration, School of Pharmacy, University of Mississippi, Mississippi, in the US from Aug 2014-July 2014. He has published several papers in journals of repute and have have presented papers in national and international conference. Additionally, he is Coordinator-Technology Transfer Office, Manipal University.

\section{REFERENCES}

1. Johnson JA, Coons SJ. Comparison of the EQ-5D and SF-12 in an adult US sample. Quality of Life Research. 1998;7(2):155-66.

2. WHOQOL Measuring Quality of Life. Division of Mental Health and Prevention of Substance Abuse. World Health Organization. 1997. WHO/MSA/MNH/ PSF/97.4.

3. Ramchandran V, Malaisamy M, Ponnaiah M. Impact of Chikungunya on Health Related Quality of Life, Chennai, South India. PLOS one. 2012;7(12). DOI: 10.1371/journal.pone.0051519.

4. Centers for Disease Control and Prevention (CDC). Health Related Quality of Life Concepts. Available from: http://www.cdc.gov/hrqol/concept.htm. [Accessed December 9, 2013].

5. Bakas T, McLennon SM, Carpenter JS. Systematic review of health related quality of life models. Health and Quality of Life Outcomes. 2012;10(1):134.

6. Guillemin F, Bombardier C, Beaton D. Cross Cultural Adaptation of Health Related Quality of Life measures: Literature Review and Proposed Guidelines. J Clin Epidemiol. 1993;46(12):1417-32.

7. Varni JW, Burwinke TM, Labe MM. Health-related quality of life measurement in pediatric clinical practice: An appraisal and precept for future research and application. Health and Quality of Life Outcomes. 2005;3(1):34.

8. Kaplan RM, Bush JW. Health-Related Quality of Life Measurement for Evaluation Research and Policy Analysis. Health Psychology. 1982;1(1):61-80.

9. Weinberger M, Oddone EZ, Samsa GP. Are Health-Related Quality-of-Life Measures Affected by the Mode of Administration?. Journal of Clinical Epidemiology. 1996;49(2):135-140.

10. Guyatt GH, Feeny DH, Patrick DL. Measuring Health-related Quality of Life. Annals of Internal Medicine. 1993;118(8):622-29.

11. Johnson JA, Pickard SA. Comparison of the EQ-5D and SF-12 in a General Population Survey in Alberta, Canada. Institute of Pharmaco-Economics Working Paper 98-6.
12. Wyrwich KW, Wolinsky FD. Identifying meaningful intra-individual change standards for health-related quality of life measures. Journal of Evaluation in Clinical Practice. 2000;6(1):39-49.

13. Cummins RA. Moving from the quality of life concept to a theory. Journal of Intellectual Disability Research. 2005;49(10):699-706.

14. Guttling JJ, de Man RA, Busschbach JJV. Overview of research on health-related quality of life in patients with chronic liver disease. The Netherlands Journal of Medicine. 2007;65(7):227-34

15. Farooqui M, Hassali MA, Knight A, et al. Cross Sectional Assessment of Health Related Quality of Life (HRQoLO among Patients with Cancer in Malaysia. Asian Pacific Journal of Cancer Prevention. 2013;14(5):3017-21.

16. Aloba O, Fatoye O, Mapayi B. A review of quality of life studies in Nigerian patients with psychiatric disorders. African Journal of Psychiatry. 2013;16(5):333-37.

17. Tian-hui C, Lu L, Michael MC. A systematic review: How to choose appropriate health-related quality of life (HRQOL) measure in routine general practice? Journal of Zhejian University Science. 2005;6B(9):936-40.

18. Zhang XH, Li SC, Fong KY. The impact of Health Literacy on Health-Related Quality of Life (HRQoL) and Utility Assessment among Patients with Rheumatic Diseases. Value in Health. 2009;12(3):S106-S9.

19. Spiegel B. Understanding Quality of Life Impact of Functional Gastrointestinal Disorders. 2007. International Foundation for Functional Gastrointestinal Disorders.

20. Rouette J. Health Related Quality of Life Outcomes from Cancer Clinical Trials: What do Oncologists Across the Globe Think? CSEB Abstract. J Epidemiol Community Health. 2013;67(10):e2.

21. Crosby RD, Kolotkin RL, Williams GR. Defining clinically meaningful change in health-related quality of life. Journal of Clinical Epidemiology. 2003;56(5):395407.

22. Cramer JA. Principles of Health-related Quality of Life: Assessment in Clinical 
Trials. Epilepsia. 2002;43(9):1084-95.

23. Chassany O, Holtmann G, Malagelada J. Systematic review: health related quality of life (HRQOL) questionnaires in gastro-oesophageal reflux disease. Alimentary Pharmacology and Therapeutics. 2008;27(11):1053-70.

24. Gurkova E. Issues in the Definitions of HRQoL. Journal of Nursing, Social Studies, Public Health and Rehabilitation. 2011;3-4:190-7.

25. Bottomley A, Jones D, Claassen L. Patient Reported Outcomes: Assessment and current perspectives of the guidelines of the Food and Drug Administrtion and the reflection paper of the European Medicines Agency. European Journal of Cancer. 2009;45(3):347-53.

26. Reflection Paper on the Regulatory Guidance for the Use of Health-Related Quality of Life (HRQL) measures in the Evaluation of Medicinal Products. European Medicines Agency 2005. Doc. Ref. EMEA/CHMP/EWP/139391/2004.

27. Guidance for Industry Patient-Reported Outcome Measures: Use in Medical Product Development to Support Labeling Claims-Draft Guidance. US Department of
Health and Human Services, Food and Drug Administration. February 2006.

28. Rock EP, Scott JA, Kennedy DL, et al. Challenges to Use of Health-Related Quality of Life for Food and Drug Administration Approvial of Anticancer Products. Journal of the National Cancer Institute Monographs. 2007;37:27-30.

29. Revicki DA. FDA draft guidance and health-outcomes research. The Lancet. 2007;369(9561):540-42

30. Aprinelli F, Bamfi F. The FDA guidance for industry on PROs: the point of view of a pharmaceutical company. Health and Quality of Life Outcomes. 2006;4(1):85.

31. Guidance for Industry Patient-Reported Outcome Measures: Use in Medical Product Development to Support Labeling Claims. US Department of Health and Human Services, Food and Drug Administration. December 2009.

32. XII Plan Document (2012-2017). Department of Health Research, Ministry of Health and Family Welfare, Government of India. 2012. Available from: http:// dhr.gov.in/madate.htm. [Accessed December 12, 2013]. 